\title{
Parametric analysis of some tensegrity structures
}

\author{
Wojciech Gilewski ${ }^{1}$, Joanna Kłosowska ${ }^{2 *}$, Paulina Obara $^{2}$ \\ ${ }^{1}$ Politechnika Warszawska, Wydział Inżynierii Lądowej, Al. Armii Ludowej 16, 00-637 Warszawa, Poland \\ ${ }^{2}$ Politechnika Świętokrzyska, Wydział Budownictwa i Architektury, Al. Tysiąclecia PP 6, 25-314 Kielce, Poland
}

\begin{abstract}
The objective of the paper are tensegrity structures and a possibility to control their properties such as a stiffness and a natural frequency, by the level of self-stress. Basic tensegrity modules and towers and plates built of these modules are considered. In each example mechanisms and self-stress states are identified using the singular value decomposition of compatibility matrix method. Parametric analyses of the effect of the self-stress state on the static and dynamic properties of structures are carried out. Analyses are performed using the second order theory (in Mathematica environment) and the third order theory (in Sofistik program).
\end{abstract}

\section{Introduction}

The objective of the paper are tensegrity structures [1-5]. The concept of such structures concerns on specific trusses which consist of compression components (struts) and tension components (cables). An important benefit of tensegrity structures is the fact that infinitesimal mechanisms are stabilized by self-stress state. The stiffness of these structures depends on the self-stress level $[1,3,4]$ and also on applied external forces.

The tensegrity concept has found applications within civil engineering, including towers and plates. There are some examples of tensegrity towers, like: Kenneth Snelson's structures (Needle Tower and Needle Tower II), Santiago Antena Tower, the tower designed by Aurel von Richthofen in Dubai, Tensegrity Barcelona Tower, the tensegrity tower by Fagerström in Tokyo and the tower designed by Ondrej Otypka [5]. Another project is Warnow Tower located in Rostock in Germany [3-5]. The last mentioned tower is created by assembling Simplex modules on top of each other. Simplex is the simplest tensegrity module. Another, most common tensegrity modules are Quartex and Octahedron. These modules may be using to build the double-layer tensegrity plate.

Double-layer tensegrity grids are one of the widely investigated applications of tensegrity structures. The first studies for the design of tensegrity grids were carried out by Snelson [6], but its applications were limited. Ariel Hanaor and René Motro et al. [7, 8] were precursors in the research of double-layer grids. For the past few years, the main focus has been in the development of double-layer tensegrity in the field of walls, roofs and covering structures $[9,10]$. The concept of creating such trusses involves the combination of tensegrity modules or modified modules by using additional connecting elements. The next researchers focused on this topic are Bin-Bing Wang, Kono, Choong, Shimada and Kunieda $[11,12]$. Their studies are connected with experimental models but there are a few examples of practical application of double-layer grids. The idea was adopted into civil engineering as the roof of a Mall inside le Meridien Hotel in Rimini. The construction of a glass roof of the National Archaeological Museum of Reggio Calabria (also known as the National Museum of Magna Graecia in Southern Italy) is the next spectacular example of tensegrity plate. Examples of applications are also the Pavilion at Expo 2002 ("Blur Building") in Switzerland and the roof of the velodrome at the World Cycling Center in Aigle, Switzerland $[9,10]$.

An important feature of tensegrity structures is the ability to control and improve their static and dynamic properties. It is possible by a controlling of self-stress forces added in truss members and by an application of external forces. The paper introduces the impact of the self-stress level on the static and dynamic behavior of the tensegrity structures. The displacements and natural frequencies are calculated [3, 13-15].

The objective of the paper is an analyze of a possibility to control properties of tensegrity structures e.g. a stiffness and a natural frequency by change the level of self-stress state.

\section{Material and methods}

The basic tensegrity modules and structures built of these modules are considered. In each example mechanisms and self-stress states are identified. To do this the distribution of the compatibility matrix according to its eigenvalues (singular value decomposition method) [16-18] is performed. To establish if the mechanism is infinitesimal the nonlinear the spectral analysis of the stiffness matrix taking into 
account the geometric stiffness matrix depended on the self-stress state, is carried out. Then, the parametric analysis of the effect of the self-stress state on the static and dynamic behavior of structures is carried out. The analysis is performed using the second order theory and the third order theory. In the first case calculations are made in Mathematica environment. In the second commercial program Sofistik to accomplish the fully geometrical non-linear analysis is used.

\subsection{Singular Value Decomposition}

The singular value decomposition (SVD) of compatibility matrix allows to determine mechanisms and self-stress states in the truss. The SVD decomposition for the compatibility matrix $\mathbf{B}$ can be expressed as following:

$$
\mathbf{B}=\mathbf{Y N X}^{T}
$$

where $\mathbf{Y}$ is orthogonal matrix consisting of eigenvectors of the matrix $\mathbf{B B}^{\mathrm{T}}, \mathbf{X}$ is orthogonal matrix consisting of eigenvectors of the matrix $\mathbf{B}^{\mathrm{T}} \mathbf{B}$ and $\mathbf{N}$ is rectangular diagonal matrix, which contains the square roots of eigenvalues of both products of matrices. Zero eigenvalues presented in matrix $\mathbf{N}$ are responsible for the existence of the self-stress state defined by the eigenvector of $\mathbf{B B}^{\mathrm{T}}$ corresponding to this value and for the existence of the mechanism defined by the eigenvector of $\mathbf{B}^{\mathrm{T}} \mathbf{B}$ corresponding to this value.

\subsection{Parametric static analysis}

The parametric analysis of the effect of the self-stress state on the static behavior of structures is carried out. The displacements are calculated. Analyses are performed using the second order theory:

$$
\left(\mathbf{K}_{L}+\mathbf{K}_{N L}^{I}\right) \mathbf{q}=\mathbf{P}
$$

and the third order theory:

$$
\left(\mathbf{K}_{L}+\mathbf{K}_{N L}^{I}+\mathbf{K}_{N L}^{I I}\right) \mathbf{q}=\mathbf{P}
$$

where $\mathbf{q}$ is the displacement vector and $\mathbf{P}$ is the load vector, $\mathbf{K}_{L}$ is the linear stiffness matrix, $\mathbf{K}_{N L}^{I}$ is the prestress stiffness matrix called the geometric stiffness matrix and $\mathbf{K}_{N L}^{I I}$ is the initial strain matrix. Stiffness matrices are determined according to the formalism of the finite element method but determination of the geometric stiffness matrix is possible if the self-stress exists.

The second order theory (2) does not include the influence of external loads on the stiffness of the structure. In order to take account the effect of additional compression the third order theory (3) should be used.

\subsection{Parametric dynamic analysis}

The parametric analysis of the effect of the self-stress state on the dynamic behavior of structures is carried out. The natural frequencies are calculated. For the case of free vibrations of the structure a generalized own problem is used:

$$
\left(\mathbf{K}_{L}+\mathbf{K}_{N L}^{I}+\omega^{2} \mathbf{M}\right) \mathbf{q}=\mathbf{0}
$$

where $\omega$ is the angular frequency and $\mathbf{M}$ is the mass matrix. The mass matrix can be constructed by variationally consistent (consistent mass) or diagonal (lumped mass) matrix. In the paper the consistent mass matrix, which establishes a uniform convergence property, is used.

Results of dynamic parametric analyses are presented as the natural frequency:

$$
f=\omega / 2 \pi
$$

and as the square of the natural frequency.

\section{Result and discussion}

Three types of the most common basic tensegrity modules are analyzed: three strut module - Simplex (Fig. 1a), four strut module - Quartex (Fig. 1b) and Octahedron (Fig. 1c). In addition, the parametric analysis of structures constructed as a result of a combining basic modules is carried out. It is the tower consisting of two (Fig. 2a) and three (Fig. 2b) Simplex modules, the plate consisting of four connected Quartex modules (Fig. 3), and the plate consisting of four Octahedron modules (Fig. 4). In order to make it possible to compare results it is assumed that all basic modules fit into a cube with $1 \mathrm{~m}$ edges. In Figures 1-4 struts and cables are drawn as thick and thin lines, respectively.

Models are supported in lower surface in such a way that the number of elements and the number of non-zero degrees of freedom are the same. Thanks to this the compatibility matrix $\mathbf{B}$ is a square matrix. In the last example (the plate consisting of four Octahedrons), the number of all degrees of a freedom is equal to the number of elements of the plate. In this case plate is supported by eight constraints and the compatibility matrix $\mathbf{B}$ is a rectangular matrix. a)

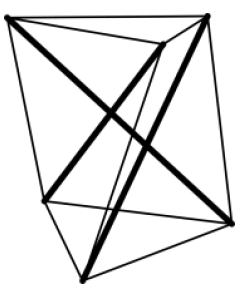

b)

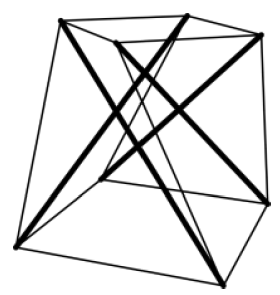

c)

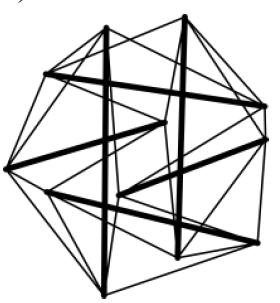

Fig. 1. Tensegrity modules: a) Simplex, b) Quartex, c) Octahedron. 
a)

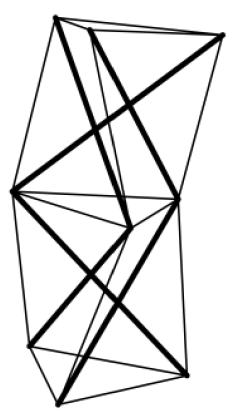

b)

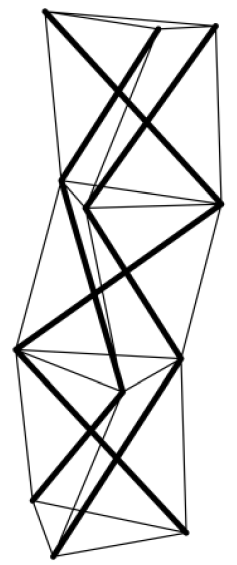

Fig. 2. Tower consisting of: a) two Simplex modules, b) three Simplex modules.

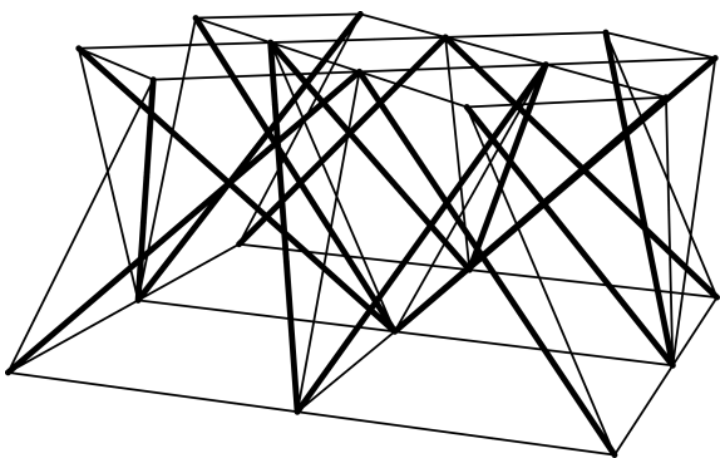

Fig. 3. Plate consisting of four Quartex modules.

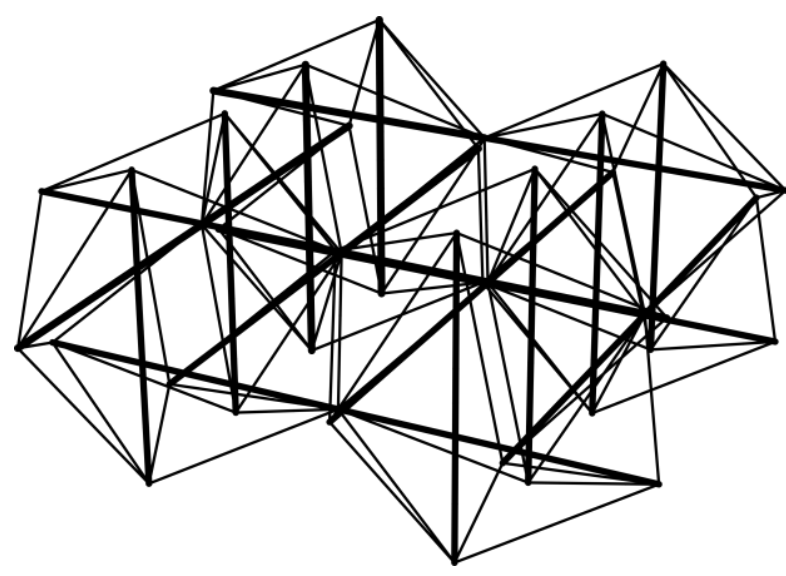

Fig. 4. Plate consisting of four Octahedron modules.

In calculations the Halfen Detan system solutions were chosen and the following mechanical and geometric properties are adopted:

- Young's modules $E=210 \mathrm{GPa}$,

- mass density $\rho=7860 \mathrm{~kg} / \mathrm{m}^{3}$,

- cables made of S460N steel: $\phi 20 \mathrm{~mm}, I=7.85 \cdot 10^{-9} \mathrm{~m}^{4}$, $A=3.1410^{-4} \mathrm{~m}^{2}$, load capacity $N_{R d}=110.2 \mathrm{kN}$,

- struts made of S355J2 steel: $\phi 76 \mathrm{~mm}$ with thickness $t=29 \mathrm{~mm}, \quad I=4.47 \cdot 10^{-7} \mathrm{~m}^{4}, \quad A=6.88 \cdot 10^{-4} \mathrm{~m}^{2}, \quad$ load capacity $N_{b, R d}=-160 \mathrm{kN}$.

For all models values of the self-stress state (determining by using the singular value decomposition) are normalized to the value -1 in struts. The following selfstress states are assumed:
- in the case of Simplex module and towers consisting of Simplex modules:

- horizontal cables: $0.3853 \cdot S$,

- diagonal cables: $0.6966 \cdot S$,

- struts: $-1 \cdot S$,

- in the case Quartex module and the plate consisting of Quartex modules:

- upper horizontal cables: $0.4714 \cdot S$,

- lower horizontal cables: $0.3333 \cdot S$,

- diagonal cables: $0.7453 \cdot S$,

- struts: $-1 \cdot \mathrm{S}$,

- in the case of Octahedron module and the plate consisting of Octahedron modules:

- cables: $0.4082 \cdot S$,

- struts $-1 \cdot S$.

In all complex structures (towers and plates), elements which are common to neighboring modules are compressed using a double value of self-stress state. When introducing prestressing forces, $80 \%$ of the load capacity of the most stressed components is not exceeded.

\subsection{Singular Value Decomposition}

For all models mechanisms and self-stress states are determined using the singular value decomposition of the compatibility matrix. The number of mechanisms and self-stresses are presented in the Table 1.

Table 1. Number of nodes, elements, self-stress states $(S S)$ and mechanisms $(M)$.

\begin{tabular}{|c|c|c|c|c|}
\hline Element & $\begin{array}{c}\text { No. of } \\
\text { nodes }\end{array}$ & $\begin{array}{c}\text { No. of } \\
\text { elements }\end{array}$ & $\begin{array}{c}\text { No. of } \\
\text { SS }\end{array}$ & $\begin{array}{c}\text { No. of } \\
\boldsymbol{M}\end{array}$ \\
\hline Simplex & 6 & 12 & 1 & 1 \\
\hline Quartex & 8 & 16 & 1 & 1 \\
\hline Octahedron & 12 & 30 & 1 & 1 \\
\hline $\begin{array}{c}\text { Tower consisting } \\
\text { of two Simplex } \\
\text { modules }\end{array}$ & 9 & 21 & 2 & 2 \\
\hline $\begin{array}{c}\text { Tower consisting } \\
\text { of three Simplex } \\
\text { modules }\end{array}$ & 12 & 30 & 3 & 3 \\
\hline $\begin{array}{c}\text { Plate consisting of } \\
\text { four Quartex } \\
\text { modules }\end{array}$ & 21 & 56 & 4 & 4 \\
\hline $\begin{array}{c}\text { Plate consisting of } \\
\text { four Octahedron } \\
\text { modules }\end{array}$ & 40 & 120 & 9 & 1 \\
\hline
\end{tabular}

\subsection{Parametric static analysis}

The parametric analysis of the effect of the self-stress state on the structure response in the field of static analysis are carried out. The models are loaded with concentrated vertical forces $P=10 \mathrm{kN}$. Singular modules are loaded with one force applied in one of the upper nodes. The extended models are loaded with four concentrated forces applied in upper nodes of structures. 
The influence of the self-stress level $S$ on maximum displacements is analyzed. The analysis is performed using the second (2nd) order theory and the third (3rd) order theory. Results are presented in Figures 5-11.

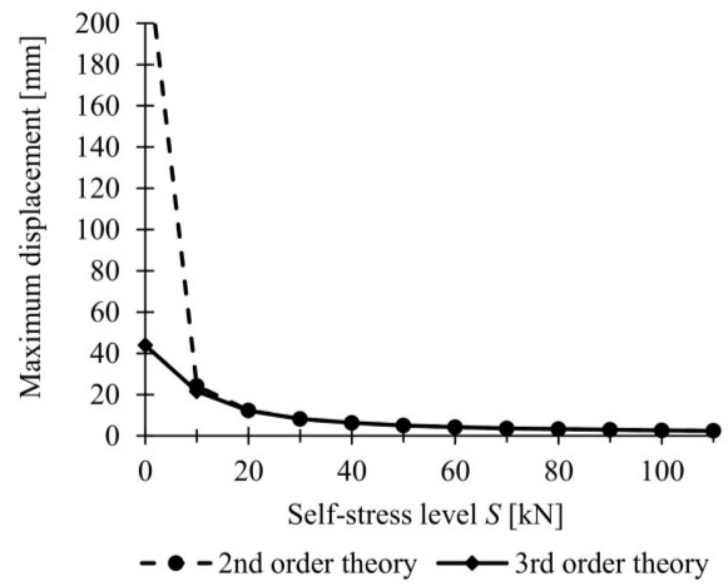

Fig. 5. Values of maximum displacement for Simplex.

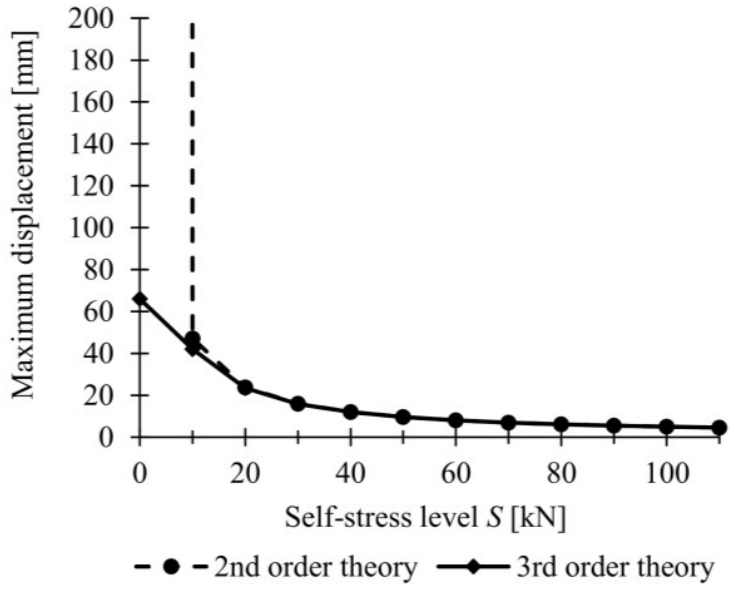

Fig. 6. Maximum displacement for Quartex.

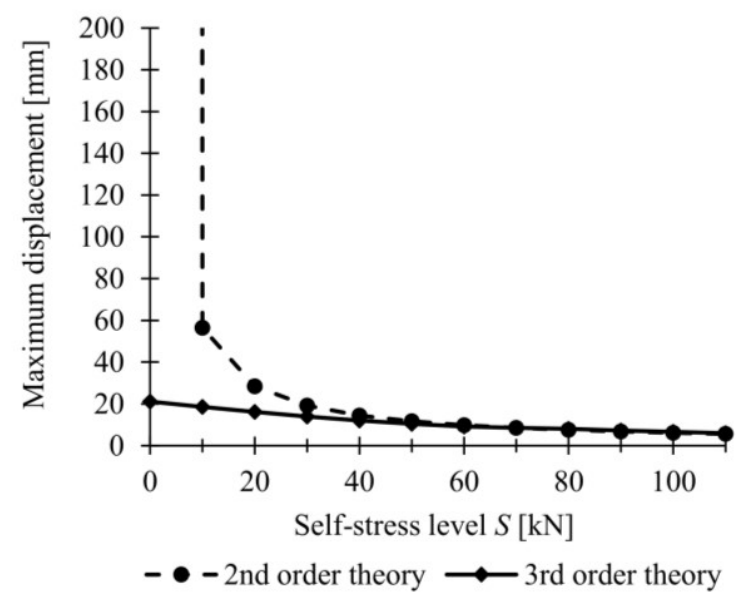

Fig. 7. Maximum displacement for Octahedron.

The Simplex module (Fig. 5) is characterized by the least sensitivity to changes of the level of self-stress. The Quartex (Fig. 6) is the most sensitive in this respect. In the case of these two modules, small differences between the results obtained according to the second and third order theory can be observed. Already at the self-stress level equal to $10 \mathrm{kN}$ results are very similar. Hence, the conclusion that in the case of these modules the influence of external loads on the stiffness is small and the second order theory is sufficient for analysis.

In the case of the Octahedron module (Fig. 7), differences between values of displacements calculated according to both theories are more noticeable. The model is more sensitive to external loads.

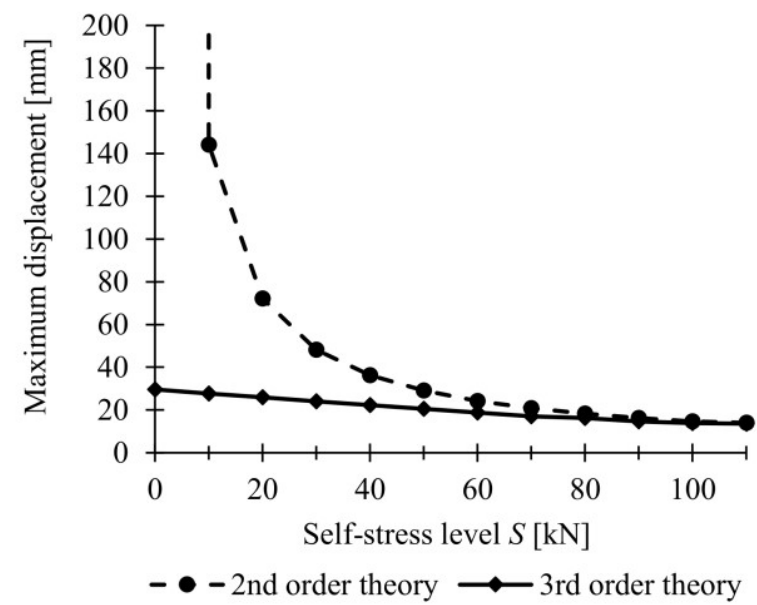

Fig. 8. Maximum displacement for the tower consisting of two Simplex modules.

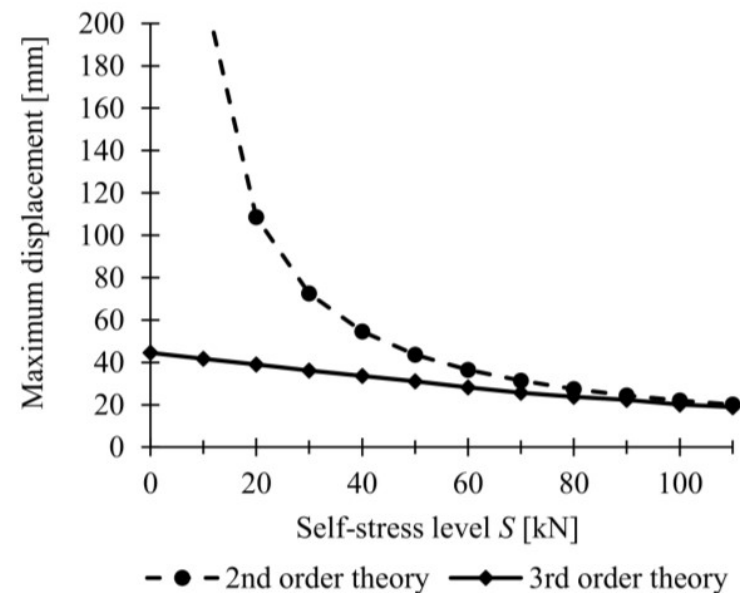

Fig. 9. Maximum displacement for the tower consisting of three Simplex modules.

Comparing results of the parametric static analysis for towers consisting of two (Fig. 8) and three Simplex (Fig. 9) it is concluded that more sensitive to external loads is second tower. It is also characterized by greater susceptibility to changes of the level of self-stress.

In the case of plates the more rigid and less sensitive to external loads is the plate consisting of four Quartex modules (Fig. 10). It is characterized by greater sensitivity to changes of the level of self-stress. In the case of the plate consisting of Octahedron modules (Fig. 11), the differences between results obtained according to the second and third order theory are significant. Values of displacements calculated according to both theories are not convergent. The plate is characterized by high sensitivity to the external load. 


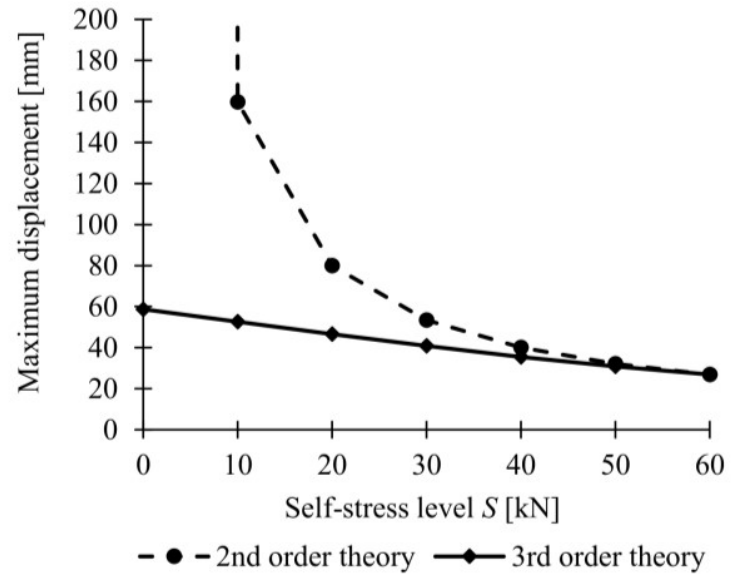

Fig. 10. Maximum displacement for a plate consisting of four Quartex modules.

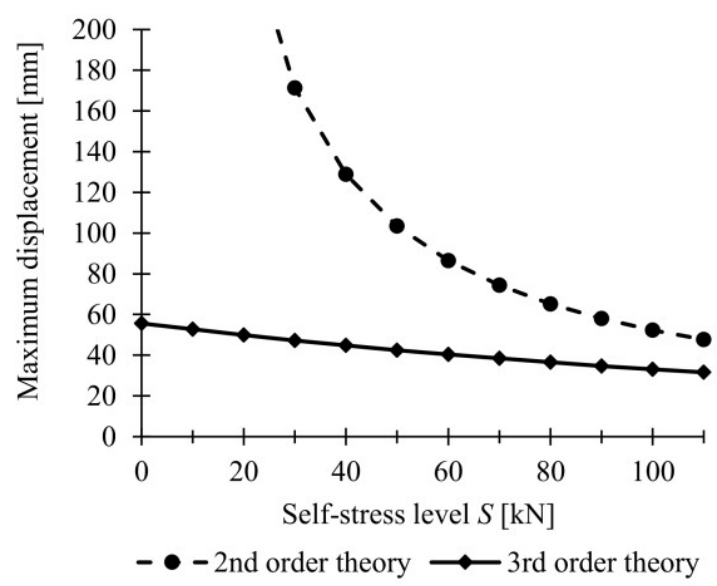

Fig. 11. Maximum displacement for a plate consisting of four Octahedron modules.

\subsection{Parametric dynamic analysis}

Parametric analyses of the effect of the self-stress state on the structure response in the field of dynamic analysis are carried out. The influence of the self-stress level $S$ on natural frequencies is analyzed. The number of natural frequencies which are dependent on the self-stress state is equal to the number of infinitesimal mechanisms. In the absence of compression, these frequencies are zero and after the introduction of the self-stress - increase. Changes of the natural frequencies, which corresponding to infinitesimal mechanisms, with increasing values of the self-stress are shown in Figures 12-15. Other natural frequencies do not change significantly with increasing the self-stress state amplitudes. For example, for basic tensegrity modules the influence of the self-stress level on the second natural frequency is shown in Table 2.

The results of the parametric dynamic analysis for basic tensegrity modules are shown in Figure 12. For Quartex and Simplex the first natural frequency is the same. The level of self-stress state has an identical impact on the natural frequency of both modules. Should be noted that Quartex is a modified module of Simplex. The third basic module - Octahedron is characterized by a lower natural frequency and less sensitivity to changes of the self-stress state on dynamic properties than previous modules. The greatest impact on the natural frequency can be observed when the level of self-stress changes from 0 to $40 \mathrm{kN}$, what corresponds to about $36 \%$ of the capacity of elements.

Table 2. Second natural frequency $f_{2}[\mathrm{~Hz}]$ for based modules.

\begin{tabular}{|c|c|c|c|}
\hline $\boldsymbol{S}[\mathbf{k N}]$ & Simplex & Quartex & Octahedron \\
\hline 0 & 127.90 & 174.81 & 87.34 \\
\hline 0.01 & 127.92 & 174.86 & 87.37 \\
\hline 10 & 127.94 & 174.91 & 87.40 \\
\hline 20 & 127.96 & 174.95 & 87.42 \\
\hline 30 & 127.98 & 175.00 & 87.45 \\
\hline 40 & 128.00 & 175.05 & 87.48 \\
\hline 50 & 128.02 & 175.09 & 87.51 \\
\hline 60 & 128.04 & 175.14 & 87.53 \\
\hline 70 & 128.05 & 175.19 & 87.56 \\
\hline 80 & 128.07 & 175.23 & 87.59 \\
\hline 90 & 128.09 & 175.28 & 87.61 \\
\hline 100 & 128.11 & 175.32 & 87.64 \\
\hline 110 & 127.90 & 174.81 & 87.34 \\
\hline
\end{tabular}

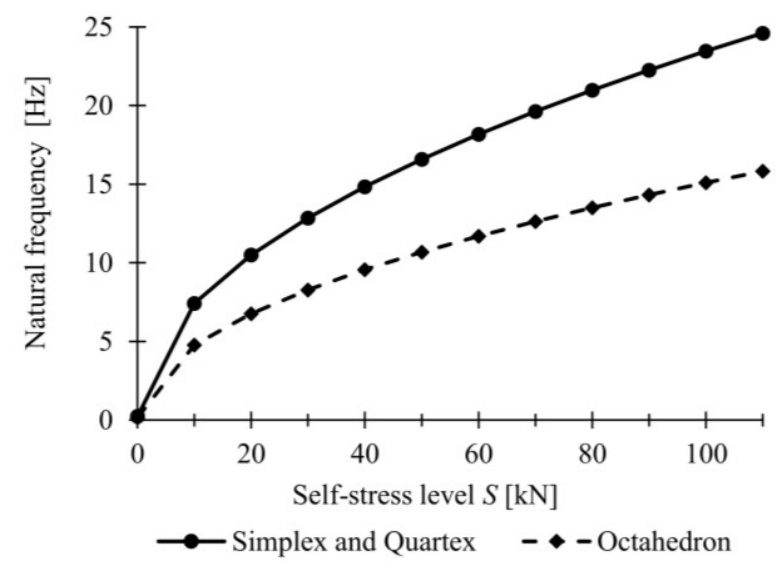

Fig. 12. First natural frequency for based modules.

Comparing results of analysis for towers (Fig. 13) it is concluded that the tower consisting of three Simplex modules is characterized by a greater sensitivity to changes of the self-stress level. Additionally, the third natural frequency of the tower consisting of three modules and the second natural frequency of the tower consisting of two modules are very similar. 


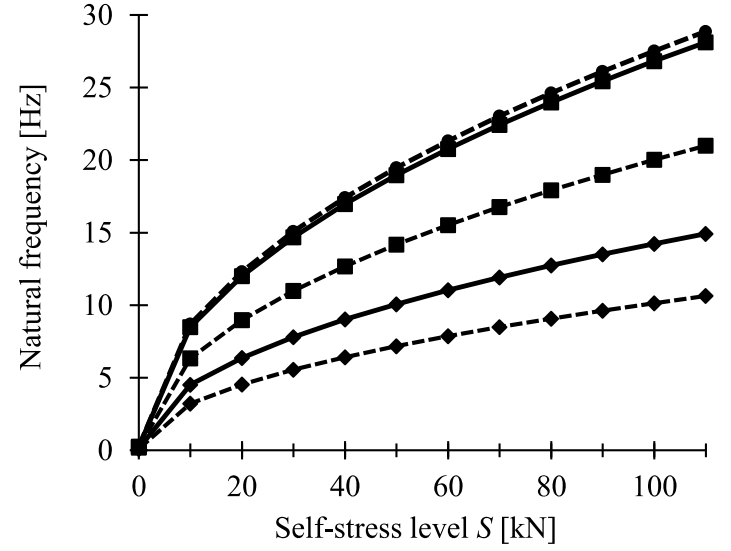

Fig. 13. Natural frequency for towers; tower consisting of two Simplex modules (-), tower consisting of three Simplex modules $(---)$; first frequency $(\bullet)$, second frequency $(\boldsymbol{\bullet})$, third frequency $(\bullet)$.

The results of the parametric dynamic analysis for plates are shown in Figures 14 and 15. In the case of the plate consisting of Quartex modules (Fig. 14), the fourth natural frequency is the most sensitive to changes of the level of self-stress. The plate consisting of Octahedron modules (Fig. 15) is characterized by the lowest natural frequency.

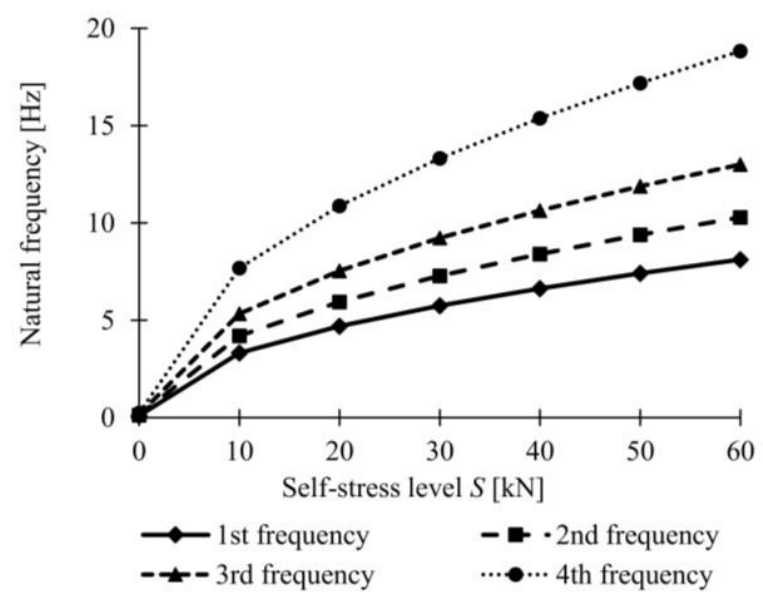

Fig. 14. Natural frequency for plate consisting of four Quartex modules.

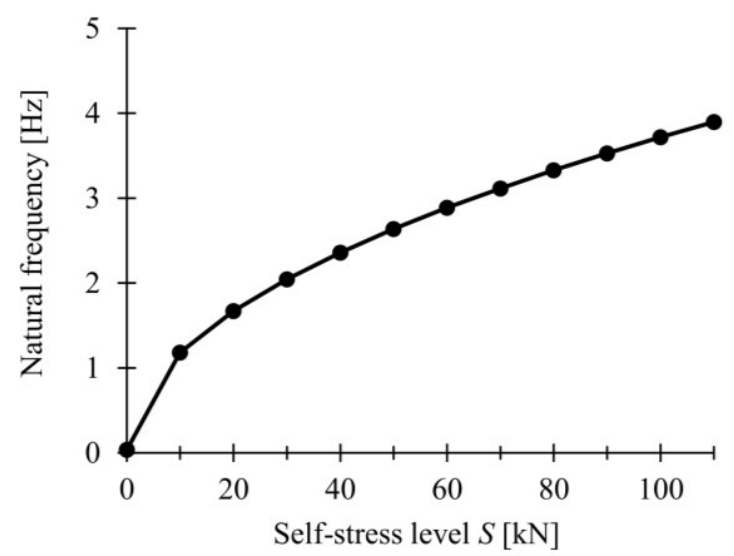

Fig. 15. First natural frequency for plate consisting of four Octahedron modules.
Additionally, the dependence between the level of self-stress state and the square of the natural frequency is examined. For all structures the square of all natural frequencies, which corresponding to infinitesimal mechanisms, increases proportionally to the amplitude of self-stress. For example, the dependence of natural frequency squared on the self-stress level for towers is presented in Figure 16.

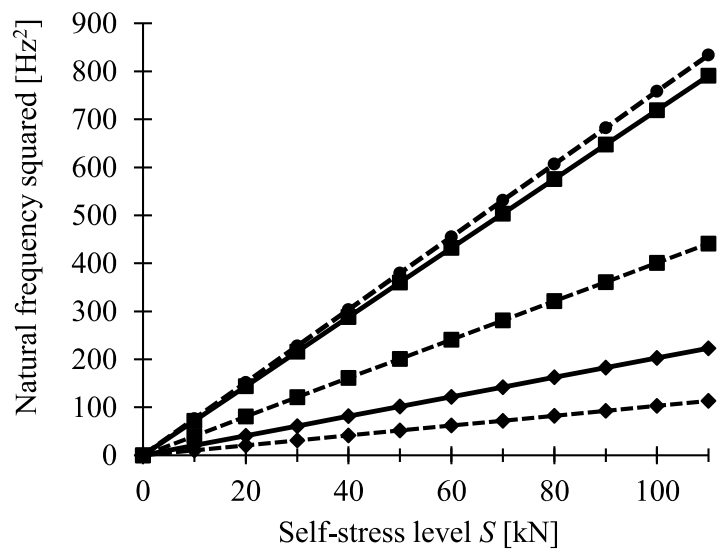

Fig. 16. Natural frequency squared for towers; tower consisting of two Simplex modules (-), tower consisting of three Simplex modules (---); first frequency $(\diamond)$, second frequency $(\boldsymbol{\bullet})$, third frequency $(\bullet)$.

\section{Conclusion}

Presented analyzes prove that tensegrity structures are distinguished by special features, which ordinary trusses do not have. It is possible to control their parameters, such as stiffness and natural frequencies, thanks to the presence of infinitesimal mechanisms and the self-stress states. For all analyzed models the influence of the selfstress level on nodal displacements is very noticeable. Nodal displacements decrease significantly with increase of internal forces of the self-stress as well as of the influence of geometrical nonlinearity is clearly seen. According to the second order theory if the value of selfstress equals zero, displacements tend to the infinity. An adjusting of prestressing forces allows to control displacements of nodes. Additionally the analysis shows that the effect of nonlinearity is the most significant at low values of self-stress forces. With increasing of selfstress differences of values of displacements calculated according to the second and the third order theory are getting smaller.

Comparing results of static analysis of tensegrity structures it is concluded that more sensitive to external loads is Octahedron module and plate consisting of these modules. In turn Simplex and Quartex module and plate consisting of Quartex modules characterize by greater sensitivity to changes of the self-stress level.

The modification of the self-stress level in tensegrity structures also allows to control their dynamic parameters. The number of natural frequencies which are dependent on the self-stress state is equal to the number of infinitesimal mechanisms. In the absence of compression, these frequencies are zero and after the 
introduction of the self-stress - increase. Other natural frequencies do not change significantly with increasing the self-stress state amplitudes.

Comparing results of dynamic analysis of tensegrity structures it is concluded that more sensitive to changes of the level of self-stress is the tower consisting of three Simplex modules.

Additionally it should be noted that the square of all natural frequencies, which corresponding to infinitesimal mechanisms, increases proportionally to the amplitude of self-stress.

\section{References}

1. B. Adam, I.F.C. Smith, Proceedings of an International Conference on Advances in Engineering Structures, Mechanics \& Construction 140, 439-448 (2006)

2. R. Motro, Tensegrity. Structural systems for the future. Kogan Page, London-Sterling (2003)

3. W. Gilewski, J. Kłosowska, P. Obara, AIP Conference Proceedings 1922, 150004 (2018)

4. W. Gilewski, A. Sabouni-Zawadzka, Arch. Civ. Eng., 60, 4, 517-534 (2014)

5. K. Snelson, Art and Ideas (2013)

6. K. Snelson, Continuous tension, discontinuous compression structures, U.S. Patent No. 3,169,611, (1965)

7. A. Hanaor, Int J Solids Struct. 8, 135-145 (1993)

8. J. Averseng, J.-F. Dube, B. Crosnier, R. Motro, Proceedings of the 44th IEEE Conference on Decision and Control, and the European Control Conference 2005, 6830-6834 (2005)

9. V. Gomez-Jauregui, R. Arias, C. Otero, C. Manchado, Int J Solids Struct. 27, 155-166 (2012)

10. A. Hanaor, Int J Solids Struct. 8, 1, 135-143 (1993)

11. B.-B. Wang, Free-standing tension structures. From tensegrity systems to cable-strut systems (2004)

12. Y. Kono, K. K. Choong, T. Shimada, H. Kunieda, IASS, 40, 103-111 (1999)

13. N. Ashwear, A. Eriksson, Comput Struct, 138, 162171 (2004)

14. J. M. Mirats-Tur, S. Hernàndez Juan, Mech. Mach. Theory, 44, 1, 1-18 (2009)

15. H. Murakami, Int J Solids Struct. 38, 3599-3613 (2001)

16. W. Gilewski, J. Kłosowska, P. Obara, Advances in Mechanics: Theoretical, Computational and Interdisciplinary Issues, 191-195 (2016)

17. S. Pellegrino, Int J Solids Struct. 30, 3025-3035 (1993)

18. V.C. Klema, A. Laub, IEEE Trans. Autom. Control 25, 164-176 (1980) 